\title{
Atrial Fibrillation Induced by Valsalva Maneuver after Cardiac Surgery
}

Yuki Nakamori ${ }^{1^{*}}$, Hiroyuki Mima ${ }^{2}$ and Kazuo Yamazaki ${ }^{2}$

${ }^{1}$ Department of Clinical Anesthesiology, Mie University Graduate School of Medicine, Tsu-city, Mie, Japan

${ }^{2}$ Department of Anesthesiology, Kobe City Medical Center General Hospital, Chuo-ku, Kobe-city, Hyogo, Japan

*Corresponding author: Yuk Nakamori, Department of Clinical Anesthesiology, Mie University Graduate School of Medicine, Tsu-city, Mie, Japan, Tel: +81592315634; E-mail: yunakamori@gmail.com

Received Date: Nov 27, 2018; Accepted Date: Dec 06, 2018; Published Date: Dec 14, 2018

Copyright: ( 2018 Nakamori Y, et al. This is an open-access article distributed under the terms of the Creative Commons Attribution License, which permits unrestricted use, distribution, and reproduction in any medium, provided the original author and source are credited.

\section{Abstract}

According to the recent guidelines, both vagal maneuvers and intravenous adenosine are recommended as the first-line treatment for paroxysmal supraventricular tachycardia (PSVT). We report a case of atrial fibrillation (AF) induced by the Valsalva maneuver performed to terminate PSVT in a male patient after cardiac surgery.

Although the efficacy of Valsalva maneuver and modified Valsalva maneuver for PSVT treatment is well known, this case suggests that these maneuvers can have a risk of inducing AF, especially after cardiac surgery. Adenosine can be an alternative strategy when the heart rate is easily controllable by epicardial pacing wires.

Keywords: Atrial fibrillation; Tachycardia; Supraventricular; Paroxysmal; Valsalva maneuver; Thoracic surgery; Postoperative complications

List of Abbreviations PSVT: Paroxysmal supraventricular tachycardia; AVNRT: Atrioventricular nodal reentrant tachycardia; AV node: Atrioventricular node; AF: Atrial fibrillation; OPCAB: Off-pump coronary artery bypass; ECG: Electrocardiogram; ICU: Intensive care unit; POAF: Postoperative atrial fibrillation; LA: Left atrium

\section{Introduction}

Tachyarrhythmia with a rapid heart rate causing cardio-respiratory failure, such as hypotension, shortness of breath, chest pain suggesting coronary ischemia, and decreased level of consciousness need acute phase treatment, except for sinus tachycardia, for which the underlying cause should be treated first.

Paroxysmal supraventricular tachycardia (PSVT) is a tachyarrhythmia caused by the reentrant circuits between the atria and the ventricles. Atrioventricular nodal reentrant tachycardia (AVNRT) is the most common type of PSVT, causing more than half of PSVT cases [1].

AVNRT involves dual electrical pathways with different conduction velocities and refractory periods within or proximal to the atrioventricular node (AV node) [2]. The ventricular rate is generally between 120 and 220 beats per minute and this fact may cause circulatory collapse.

According to the recent guidelines, both vagal maneuvers and intravenous adenosine are recommended as the first-line acute treatment of AVNRT. If these therapies cannot terminate the tachycardia and the hemodynamic status is still unstable, synchronized cardioversion is recommended [3].
Among the used vagal maneuvers, carotid sinus massage may detach intra-arterial plaques and cause neurological complications [4].

Standard Valsalva maneuver is effective for $45 \%$ of cases with AVNRT. It has no physical effects on the cervical artery and can be very easily performed at the bedside. Therefore, this maneuver can be successfully applied in the acute phase of the arrhythmia, even if the vagal maneuvers have a potential risk of transient sinus pauses or prolonged atrioventricular block [5].

We herein report a case of atrial fibrillation (AF) triggered by the Valsalva maneuver performed to terminate AVNRT in a postoperative patient who underwent off-pump coronary artery bypass (OPCAB). We believe that this is an important finding for intensivists and emergency physicians who perform acute treatment for PSVT.

\section{Case Report}

A 79-year-old male was admitted to our emergency service for palpitation. Electrocardiogram (ECG) showed narrow QRS tachycardia (192bpm) with retrograde P wave following the QRS complex. No evidence of QT prolongation nor bundle branch block was found (Figure 1). The patient's hemodynamic status was stable. He was diagnosed with PSVT and was prescribed $40 \mathrm{mg}$ of verapamil orally. Soon after taking the verapamil, his heart rate decreased to 99 bpm and his ECG showed a regular sinus rhythm (Figure 2). Ablation for PSVT was planned, but myocardial scintigraphy performed during the preoperative examination showed abnormal myocardial perfusion of the inferior and lateral wall of the left ventricle. Coronary angiography revealed a three-vessel disease. Therefore, he was scheduled for OPCAB before the ablation therapy. The degree of heart failure was New York Heart Association class I. Since the first emergency episode, he had no recurrent palpitation and his ECG had showed a regular sinus rhythm. 
Citation: $\quad$ Nakamori Y, Mima H, Yamazaki K (2018) Atrial Fibrillation Induced by Valsalva Maneuver after Cardiac Surgery. Med Rep Case Stud 3:

HR: $19210.00 \mathrm{~mm} / \mathrm{m} / 25.0 \mathrm{mu} / \mathrm{s} 100 \mathrm{~Hz} \quad 10.00 \mathrm{mu} / \mathrm{mV} 25.0 \mathrm{~mm} / \mathrm{s} \quad 100 \mathrm{~Hz}$

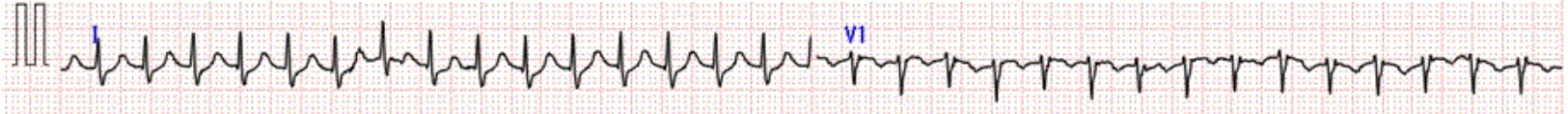

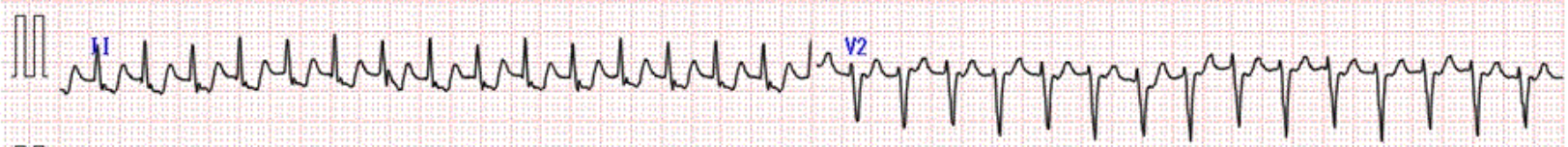

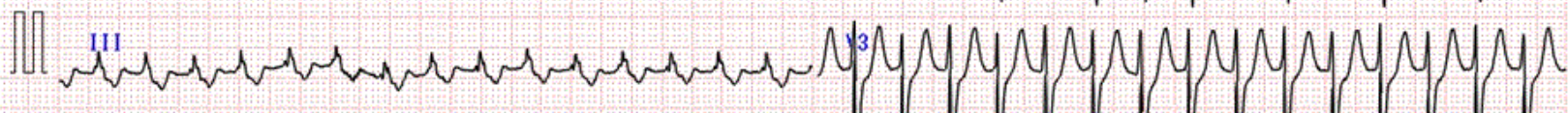

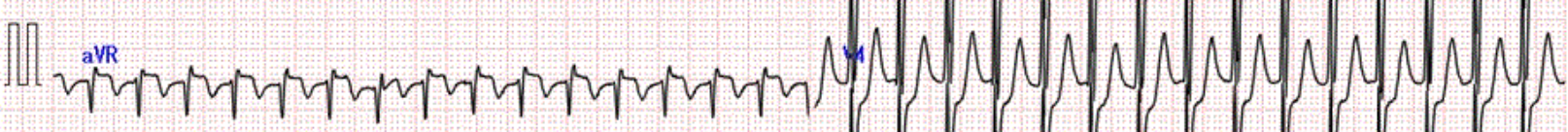

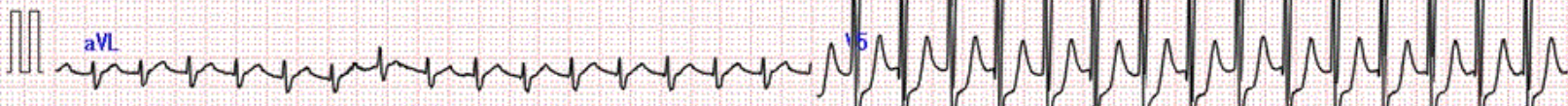

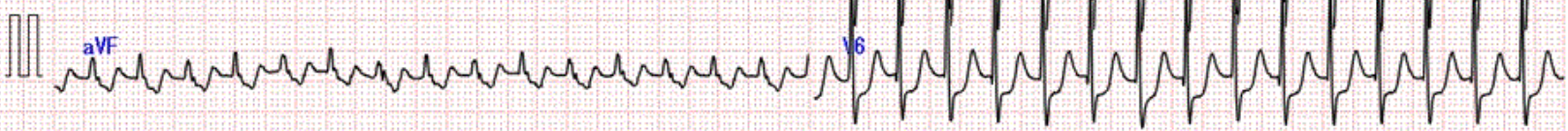

Figure 1: ECG of the patient after admission.



Figure 2: ECG showing a regular sinus rhythm.

The OPCAB operation was performed without problems until the last peripheral suture. During the posterolateral branch suture, sustained ventricular tachycardia occurred and because of the instable hemodynamic status, the cardioversion with 10J was performed. After the cardioversion, the sinus rhythm was stable and the operation was successfully finished. An epicardial pacing wire was inserted on the anterior right ventricle wall.

In the intensive care unit (ICU), the patient cardiopulmonary function was stable under the continuous dobutamine infusion at the rate of $2 \mathrm{mcg} / \mathrm{kg} / \mathrm{min}$. He was extubated in the morning of the 
postoperative day one and the inotropic agent was stopped in the afternoon of the same day.

After extubation, he was managed without sedation. In the evening of the postoperative day one, he developed two episodes of PSVT associated with chest pain, hypotension (systolic blood pressure less than $80 \mathrm{mmHg}$ ), and palpitation (Figure 3). We attempted to stop PSVT by Valsalva maneuver. The attempt was successful for the first episode, but not for the second. For the latter episode, we injected 10 $\mathrm{mg}$ of adenosine, which terminated the PSVT.

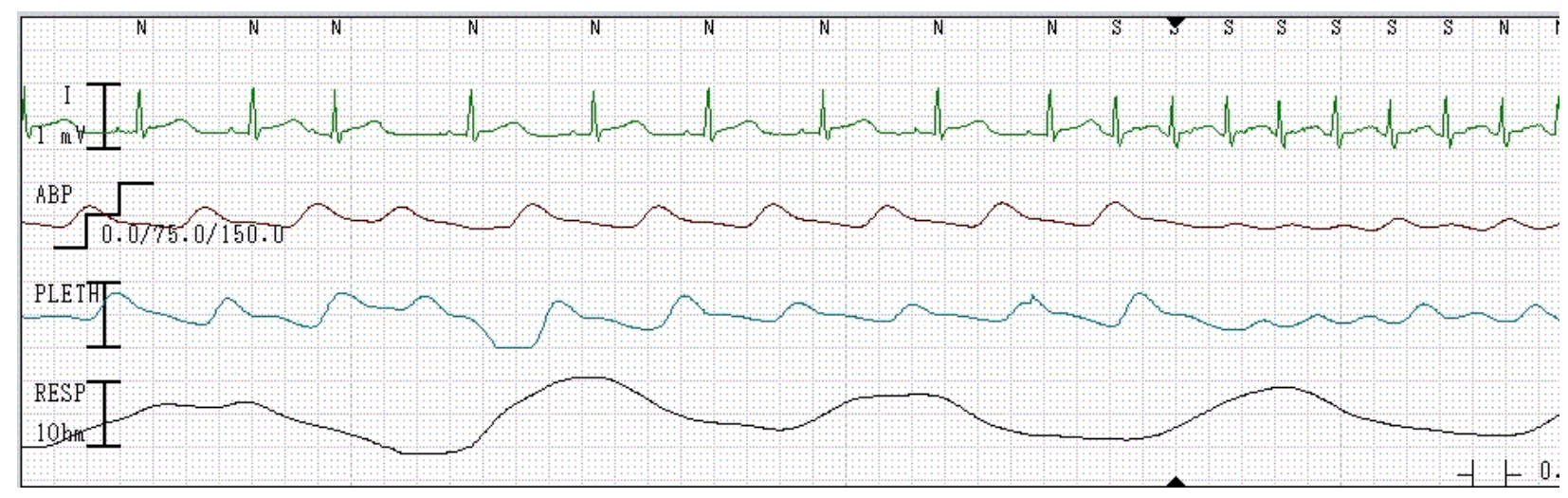

Figure 3: Two episodes of PSVT.

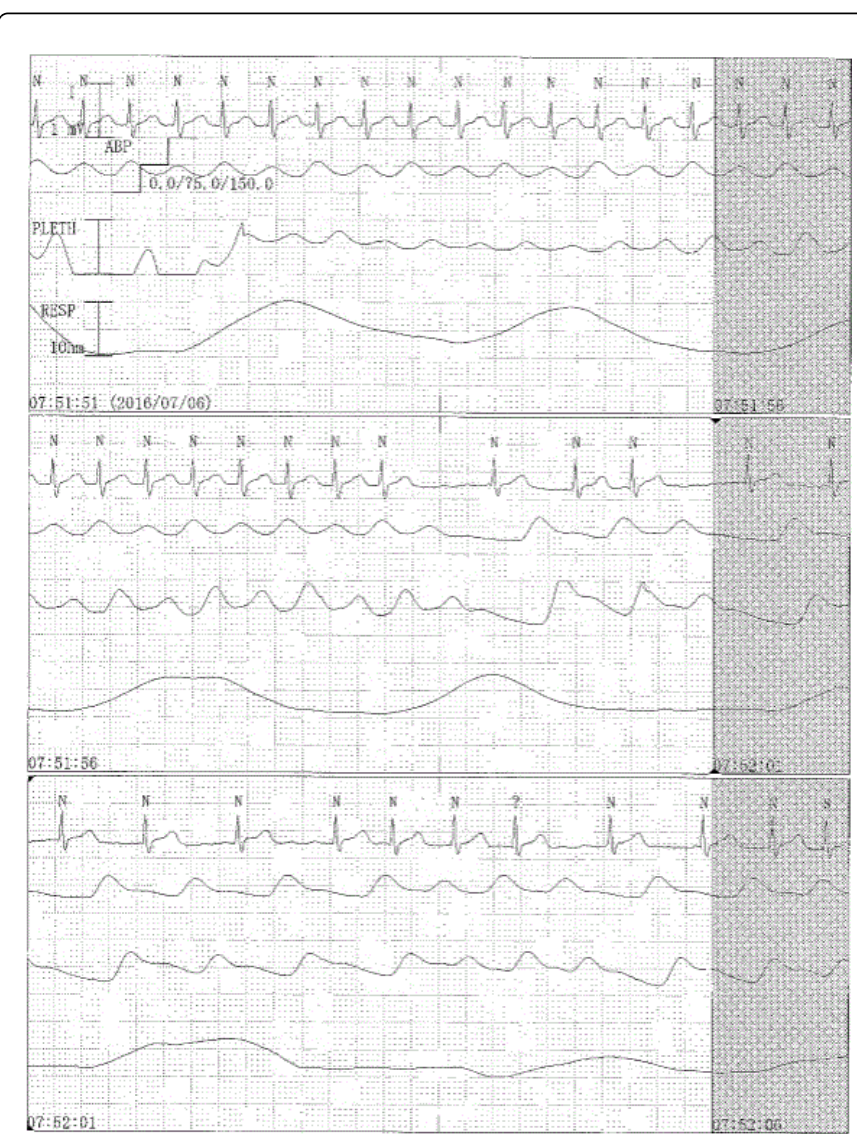

Figure 4: Rhythm degenerated into AF.

In the morning of the postoperative day two, a third episode of PSVT appeared. As in previous cases, we first asked the patient to perform Valsalva maneuver. However, this time his rhythm degenerated into AF (Figure 4). His hemodynamic parameters were relatively stable; therefore, we chose to use pharmacological defibrillation with amiodarone loading instead of electrical defibrillation. In four hours, the sinus rhythm was restored.

\section{Discussion}

This report presents a case of AF induced by Valsalva maneuver applied to terminate an episode of PSVT.

Tachycardias are broadly categorized based on the width of the QRS complex on the ECG into narrow and wide QRS complex tachycardias. This case shows a narrow QRS tachycardia, specifically supraventricular tachycardia.

In this case, the $\mathrm{P}$ wave did not precede the QRS complex. In addition, a negative $\mathrm{P}$ wave with a short RP interval followed the QRS complex. These ECG findings suggested AVNRT [6]. Although we could not rule out atrioventricular reentrant tachycardia related to a concealed WPW syndrome, the treatment strategy for each of them is similar, consisting of a transient $\mathrm{AV}$ node block induction.

As the patient complained of chest pain with the systolic BP less than $90 \mathrm{mmHg}$, we attempted early sinus node recovery. In accordance with the guidelines, vagal maneuvers were first attempted. Because the patient had an epicardial temporary pacing wire, a potential bradycardia triggered by the vagal maneuvers was not a concern. We did not perform carotid sinus massage because preoperative carotid artery ultrasonography showed atherosclerotic plaques. Dobutamine was injected for the cardiac output support a few hours before, so the use of $\beta$-blockers or calcium channel blockers to suppress AV node conduction was not appropriate. Even if rapid sinus recovery was expected, the patient was consciousness and synchronized cardioversion was withheld until all other medical therapies failed. Finally, we selected to perform conventional Valsalva maneuver, because our ICU ward beds did not allow rapid achievement of "lying down with leg lift" modified Valsalva motion [7]. 
Postoperative atrial fibrillation (POAF) is a common event after cardiac surgeries, and its incidence is approximately 30\% after coronary artery bypass grafting. Most of POAF occurs in the postoperative day 2 or 3 and more than $70 \%$ of them are reported until postoperative day $4[8,9]$.

This patient also had AF during the common phase of POAF. This case revealed that careful management is necessary for performing Valsalva maneuvers, especially in postoperative cases. Typically, we should take enough measures, such as keeping high serum potassium level, to prevent $\mathrm{AF}$ in the postoperative patients.

Valsalva maneuver usually stimulates parasympathetic nervous system. AF can be triggered not only by sympathetic nerve stimulation, but also by parasympathetic stimulation [10]. Increased parasympathetic activity precedes predominantly nocturnal AF [11].

Valsalva maneuver increases the intra-alveolar pressure and transiently forces the blood from pulmonary vascular bed into the left atrium (LA). Acute stretch of the LA results in slower conduction across the pulmonary vein-LA junction, with more signal complexity. This substrate may be important in AF initiation and maintenance by promoting the re-entry phenomenon [12].

AF could already occur in the atrium during the PSVT, because $19 \%$ of AVNRT episodes coexist with AF [13]. Therefore, we should assume that terminating PSVT might trigger concealed AF.

Because a superior efficacy of modified Valsalva maneuver for PSVT compared to the standard Valsalva maneuver was reported recently, the number of applied Valsalva maneuvers is expected to increase [7]. However, like in this case, Valsalva maneuver can potentially cause AF. Therefore, adenosine as the first-line treatment would be a reasonable strategy for the patients with available pacing, such as those after cardiac surgery.

Obviously, the routine insertion of epicardial pacing for cardiac surgery is not recommended $[14,15]$. Epicardial wires can induce many complications, including infection, myocardial damage, perforation, tamponade, and disruption of coronary anastomoses [16]. Moreover, $\mathrm{R}$ on $\mathrm{T}$ phenomenon can occur regardless of proper sense threshold setting [17]. Nevertheless, epicardial pacing insertion is good strategy for the PSVT cases detected preoperatively, for Valsalva maneuver have a potential risk of inducing $\mathrm{AF}$.

In conclusion, we report a case of AF induced by Valsalva maneuver for PSVT. Only one Hungarian paper about the reported Valsalva maneuver complication was published [18]. During the susceptible period of POAF, when blood pressure is pharmacologically maintained and $\beta$-blockers or calcium channel blocker are not appropriate, intravenous adenosine or synchronized cardioversion are reasonable strategies for terminating the PSVT. Especially when the heart rate is easily controllable by epicardial pacing wires, adenosine should be the first-line choice.

\section{Consent}

Written informed consent was obtained from the patient for publication of this Case report and any accompanying images. A copy of the written consent is available for review by the Editor-in-Chief of this journal.

\section{Financial Disclosures}

None

\section{Conflicts of Interest}

None

\section{References}

1. Ganz LI, Friedman PL (1995) Supraventricular tachycardia. N Engl J Med 332: 162-173.

2. McGuire MA, Bourke JP, Robotin MC, Johnson DC, Meldrum-Hanna W, et al. (1993) High resolution mapping of Koch's triangle using sixty electrodes in humans with atrioventricular junctional (AV nodal) reentrant tachycardia. Circulation 88: 2315-2328.

3. Page RL, Joglar JA, Caldwell MA, Calkins H, Conti JB, et al. (2015) 2015 ACC/AHA/HRS Guideline for the Management of Adult Patients With Supraventricular Tachycardia: A Report of the American College of Cardiology/American Heart Association Task Force on Clinical Practice Guidelines and the Heart Rhythm Society. J Am Coll Cardiol 67: e27e115.

4. Richardson DA, Bexton R, Shaw FE, Steen N, Bond J, et al. (2000) Complications of carotid sinus massage--a prospective series of older patients. Age Ageing 29: 413-417.

5. Pandya A, Lang E (2015) Valsalva maneuver for termination of supraventricular tachycardia. Ann Emerg Med 65: 27-29.

6. Letsas KP, Weber R, Siklody CH, Mihas CC, Stockinger J, et al. (2010) Electrocardiographic differentiation of common type atrioventricular nodal reentrant tachycardia from atrioventricular reciprocating tachycardia via a concealed accessory pathway. Acta cardiologica 65: 171-176.

7. Appelboam A, Reuben A, Mann C, Gagg J, Ewings P, et al. (2015) Postural modification to the standard Valsalva manoeuvre for emergency treatment of supraventricular tachycardias (REVERT): a randomised controlled trial. Lancet 386: 1747-1753.

8. Hogue CW Jr., Creswell LL, Gutterman DD, Fleisher LA (2005) Epidemiology, mechanisms, and risks: American College of Chest Physicians guidelines for the prevention and management of postoperative atrial fibrillation after cardiac surgery. Chest 128: 9s-16s.

9. Mitchell LB (2011) Canadian Cardiovascular Society atrial fibrillation guidelines 2010: prevention and treatment of atrial fibrillation following cardiac surgery. Can J Cardiol 27: 91-97.

10. Coumel P (1996) Autonomic influences in atrial tachyarrhythmias. J Cardiovasc Electrophysiol 7: 999-1007.

11. Herweg B, Dalal P, Nagy B, Schweitzer P (1998) Power spectral analysis of heart period variability of preceding sinus rhythm before initiation of paroxysmal atrial fibrillation. Am J Cardiol 82: 869-874.

12. Walters TE, Lee G, Spence S, Larobina M, Atkinson V, et al. (2014) Acute atrial stretch results in conduction slowing and complex signals at the pulmonary vein to left atrial junction: insights into the mechanism of pulmonary vein arrhythmogenesis. Circ Arrhythm Electrophysiol 7: 1189-1197.

13. Schernthaner C, Danmayr F, Strohmer B (2014) Coexistence of atrioventricular nodal reentrant tachycardia with other forms of arrhythmias. Med Princ Pract 23: 543-550.

14. Alwaqfi NR, Ibrahim KS, Khader YS, Baker AA (2014) Predictors of temporary epicardial pacing wires use after valve surgery. J Cardiothorac Surg 9: 33.

15. Ferrari AD, Sussenbach CP, Guaragna JC, Piccoli Jda C, Gazzoni GF, et al. (2011) Atrioventricular block in the postoperative period of heart valve surgery: incidence, risk factors and hospital evolution. Brazilian Journal of Cardiovascular Surgery 26: 364-372.

16. Reade MC (2007) Temporary epicardial pacing after cardiac surgery: a practical review: part 1: general considerations in the management of epicardial pacing. Anaesthesia 62: 264-271.

17. Nakamori Y, Maeda T, Ohnishi Y (2016) Reiterative ventricular fibrillation caused by R-on-T during temporary epicardial pacing: a case report. JA Clin Rep 2: 3 . 
Citation: $\quad$ Nakamori Y, Mima H, Yamazaki K (2018) Atrial Fibrillation Induced by Valsalva Maneuver after Cardiac Surgery. Med Rep Case Stud 3: 172. doi:10.4172/2572-5130.1000172

Page 5 of 5

18. Makai A, Zollei E, Halmai L, Rudas L (1999) Atrial fibrillation provoked by Valsalva maneuver. Orv Hetil 140: 2811-2812. 